\title{
Uma Revisão Sistemática Sobre Técnicas de Audio Games Para Promover Acessibilidade de Pessoas com Deficiência Visual no Uso de Games
}

\author{
Davi A. da Silva Ribeiro, Igor J. Richtic Silva, Thiago R. Lobo, Luciana C. Lima \\ de Faria Borges, Eunice P. dos Santos Nunes
}

Instituto de Computação - Universidade Federal de Mato Grosso (UFMT)

CEP 78.060-900 - Cuiabá - MT - Brasil

davi.ribeiro@aluno.ic.ufmt.br, igorjordanyegmail.com,

thiago.ruiz.trl@gmail.com, lucianafariaborges@gmail.com, eunice@ufmt.br

\begin{abstract}
Resumo. Este artigo apresenta os resultados preliminares de uma Revisão Sistemática sobre técnicas de Audio Games, utilizadas na acessibilidade de Pessoas com Deficiência (PcD) visual em Ambientes Virtuais, especificamente, Games. As técnicas de áudio games buscam promover a interação e imersão da PcD visual nos games, e os resultados mostraram que é possível implementar games acessíveis a PcD visual em diferentes contextos.
\end{abstract}

\section{Introdução}

De modo geral, o processo de desenvolvimento de games envolve a junção de diversas áreas da computação como, por exemplo, Realidade Virtual, Realidade Aumentada, Computação Gráfica, Games e Inteligência Artificial. Entretanto, o processo de desenvolvimento de games exige adotar uma metodologia que vise garantir a qualidade do produto final.

$\mathrm{Na}$ literatura, encontram-se diversos processos de desenvolvimento de games, entre eles o processo apresentado no trabalho de Cuzziol (2007), com três etapas principais: a) concepção, no qual se tem o detalhamento do game, ou seja, o roteiro do game, no qual consta fichas de personagens, concepção de ambientes, efeitos sonoros, música, interface, fluxo do game, comportamento artificial; b) produção, que inclui o desenvolvimento e junção dos componentes do game, como o design do cenário, design de som, design gráfico, modelagem, texturização, animação e programação; c) integração, que inclui as etapas de testes e avaliação com potenciais usuários.

Nesse contexto, este trabalho está focado no componente que trata do design de som, acessível às Pessoas com Deficiência (PcD) visual. De acordo com Horowitz e Looney (2014), design de som é qualquer forma de manipulação sonora dentro do projeto de games, desde criação de som, gravação, edição e aplicação em um game Apesar dos avanços na área de design de som nos últimos 10 anos, o desenvolvimento de games acessíveis à pessoa com deficiência visual ainda é incipiente [Östblad et al. 2014]. 
Considerando um bom modelo de design de som, que atenda a acessibilidade de pessoas com deficiência visual nos games, encontra-se na literatura o conceito de audio games, que de acordo com Rovithis et al. (2015), são games que dependem principalmente ou exclusivamente do áudio. As interfaces auditivas são usadas para interação e percepção do ambiente, logo os jogadores necessitam usar o sentido auditivo para percepção e imersão no mesmo. Rovithis et al. (2015) definem audio games em dois tipos: a) Audio Only Games - não possui interface gráfica para interação, sendo a interação exclusivamente por meio de áudio; e b) Audio Based Games - o áudio é a base do game, mas com interface gráfica para interação. Em ambos os tipos de audio games, o áudio é a principal ferramenta de interação humano-computador.

Considerando a inclusão de PcD no mundo virtual dos games, especificamente deficiência visual, este trabalho apresenta os resultados preliminares de uma Revisão Sistemática (RS) sobre audio games, que buscou investigar técnicas e ferramentas que possam ser utilizadas na produção de um game acessível.

\section{Metodologia}

A RS conduzida seguiu recomendações da metodologia PRISMA [Moher et al. 2009], e apresenta três fases: planejamento, condução e extração de resultados. A RS teve o propósito de responder a seguinte questão de pesquisa: Quais estratégias de interação e técnicas de áudio têm sido aplicadas em Ambientes Virtuais ou games, a fim de torná-los acessíveis a Pessoas com Deficiência visual?

$\mathrm{Na}$ etapa de planejamento, foi pré-estabelecido o protocolo de RS, definindo diretrizes que foram seguidas ao longo da revisão como: questão de pesquisa, palavraschave, bases de dados científicas nas quais as strings de buscas foram aplicadas, os critérios de inclusão e exclusão de artigos e uma breve descrição dos procedimentos que aplicados nas fases da RS.

Na etapa de condução, procurou-se por estudos primários nos últimos cinco anos, a fim de encontrar novas abordagens. A etapa de condução da RS considerou duas fases importantes: i) seleção preliminar, que consistiu na análise dos títulos e resumos de cada trabalho; e ii) seleção final, que consistiu na análise dos textos completos dos artigos incluídos na fase de seleção preliminar. A seleção dos trabalhos de interesse foi realizada de acordo com os critérios de inclusão e exclusão definidos no protocolo.

E por fim, na etapa de extração de resultados, os estudos selecionados foram investigados, a fim de entender o estado da arte na área que está sendo investigada.

A Figura 1 apresenta o fluxograma das diferentes fases da RS, que buscou responder à questão de pesquisa, combinando-se as strings de busca a partir das palavraschave e aplicadas nas bases de dados IEEE e ACM. 


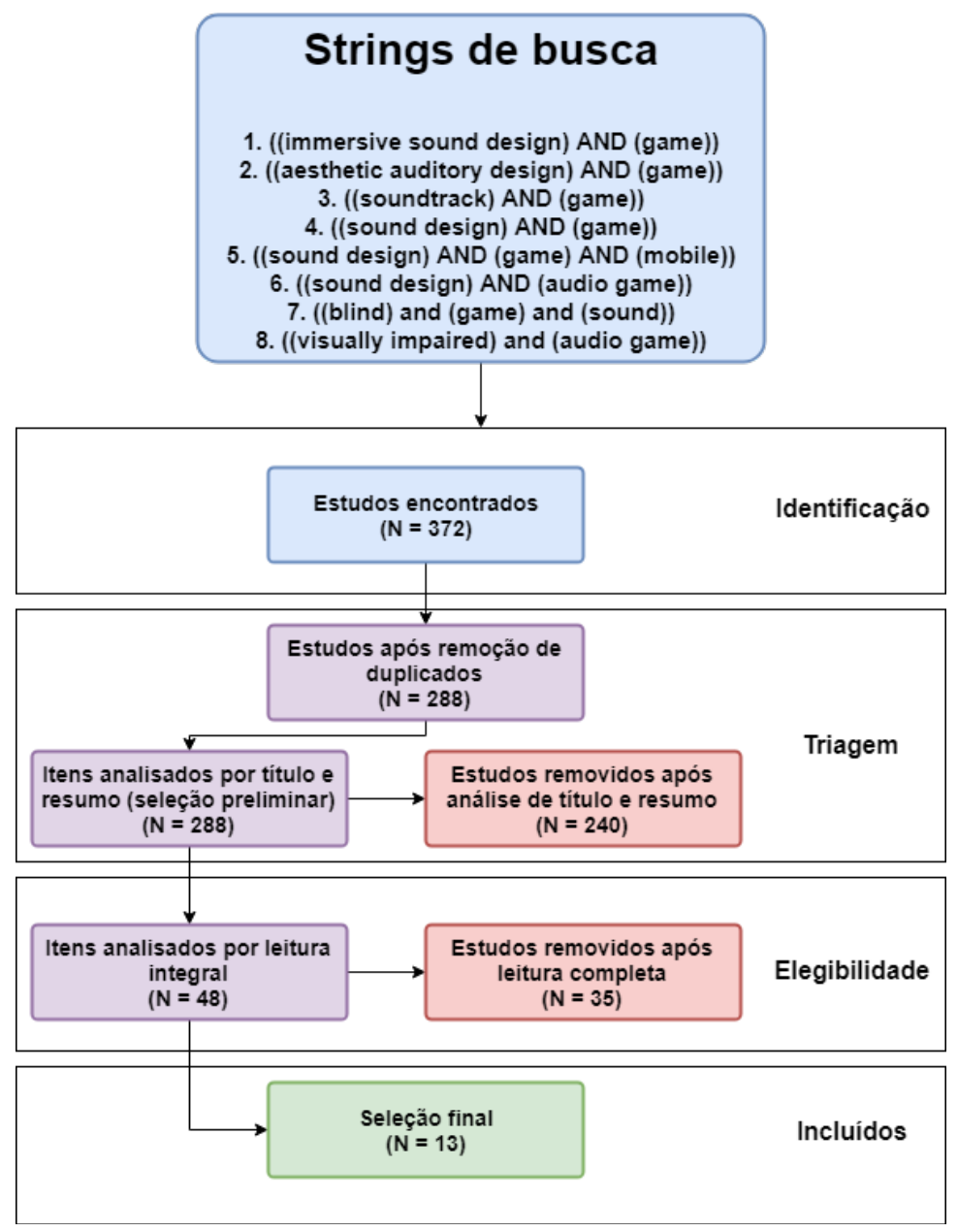

Figura 1 - Fluxograma da Revisão Sistemática

Observa-se na Figura 1 que 372 trabalhos foram encontrados no processo de RS, os quais passaram por uma etapa de triagem, eliminando estudos duplicados e posterior a isso, foi feita uma seleção preliminar baseada no título e resumo dos trabalhos, descartando-se 240 estudos. Na seleção final, 48 estudos foram lidos na íntegra, que após aplicados os critérios de inclusão e exclusão foram selecionados ao final 13 trabalhos que apresentaram respostas às questões de pesquisa, e dos quais foram extraídos os resultados que são apresentados na seção 3 .

\section{Resultados da Revisão Sistemática}

Os estudos selecionados na RS simbolizam o primeiro passo de um estudo que faz parte de um contexto maior, que é conceber e avaliar Ambientes Virtuais Tridimensionais (AVs 3D) acessíveis a PcD visual.

A Tabela 1 apresenta uma síntese dos resultados obtidos com os 13 trabalhos incluídos na RS, ordenados por ano de publicação. Os principais aspectos extraídos são: abordagem do AV, desafio a ser superado pelo usuário, tipo de som usado no AV, plataforma, quais dispositivos de interação são usados e se houve realização de experimentos. 
Tabela 1. Principais aspectos dos artigos selecionados na RS

\begin{tabular}{|c|c|c|c|c|c|c|}
\hline Fonte & Abordagem & Desafio & $\begin{array}{c}\text { Tipo de } \\
\text { som }\end{array}$ & Plataforma & \begin{tabular}{|c|} 
Interação \\
com \\
dispositivo
\end{tabular} & Experimento \\
\hline $\begin{array}{l}\text { [Pires, } \\
\text { Furtado e } \\
\text { Carregã } \\
\text { 2013] }\end{array}$ & $\begin{array}{c}\text { Treinamento de } \\
\text { orientação } \\
\text { espacial }\end{array}$ & $\begin{array}{c}\text { Encontrar } \\
\text { narrativas e } \\
\text { completá-las }\end{array}$ & $\begin{array}{l}\text { diálogos, } \\
\text { foley, 3D } \\
\text { ambiente } \\
\text { e música }\end{array}$ & computador & Teclado & $\mathrm{X}$ \\
\hline $\begin{array}{c}{[\text { Ferreira e }} \\
\text { Cavaco 2014] }\end{array}$ & $\begin{array}{l}\text { Ensino de } \\
\text { matemática }\end{array}$ & $\begin{array}{c}\text { Responder } \\
\text { questões de } \\
\text { matemática e } \\
\text { explorar }\end{array}$ & $\begin{array}{l}\text { diálogos, } \\
\text { foley, 2D } \\
\text { ambiente } \\
\text { e música }\end{array}$ & computador & Teclado & $\mathrm{X}$ \\
\hline $\begin{array}{c}\text { [Rovithis, } \\
\text { Mniestris e } \\
\text { Floros 2014] }\end{array}$ & $\begin{array}{c}\text { Entretenimento } \\
\text { inclusão e } \\
\text { game RPG }\end{array} \mid$ & $\begin{array}{c}\text { Explorar } \\
\text { cenário e } \\
\text { seguir } \\
\text { narrativa de } \\
\text { RPG. }\end{array}$ & $\begin{array}{l}\text { diálogos, } \\
\text { foley, 3D } \\
\text { ambiente }\end{array}$ & computador & - & - \\
\hline $\begin{array}{c}\text { [Simões e Ca } \\
\text { vaco 2014] }\end{array}$ & $\begin{array}{c}\text { Treinamento de } \\
\text { orientação } \\
\text { espacial }\end{array}$ & $\begin{array}{l}\text { Identificar } \\
\text { direção de } \\
\text { insetos } \\
\text { alienígenas }\end{array}$ & \begin{tabular}{|c|} 
foley, 3D \\
ambiente, \\
efeitos \\
sonoros
\end{tabular} & celular & Touchscreen & $\mathrm{X}$ \\
\hline $\begin{array}{c}\text { [Balan et al., } \\
\text { 2014] }\end{array}$ & $\begin{array}{c}\text { game sério para } \\
\text { treinamento de } \\
\text { orientação } \\
\text { espacial }\end{array}$ & $\begin{array}{c}\text { localizar } \\
\text { objeto } \\
\text { sonoro em } \\
\text { um ambiente }\end{array}$ & $\begin{array}{c}\text { 3D } \\
\text { ambiente, } \\
\text { efeitos } \\
\text { sonoros }\end{array}$ & computador & mouse & $\mathrm{X}$ \\
\hline $\begin{array}{c}\text { [Kim e Baek } \\
\text { 2014] }\end{array}$ & \begin{tabular}{|c|} 
Treinamento \\
orientação \\
espacial e \\
entretenimento
\end{tabular} & $\begin{array}{c}\text { labirinto com } \\
\text { obstáculos } \\
\text { sonoros }\end{array}$ & \begin{tabular}{|} 
3D \\
ambiente, \\
efeitos \\
sonoros, \\
foley, \\
música
\end{tabular} & computador & teclado & - \\
\hline $\begin{array}{l}\text { [Östblad et } \\
\text { al. 2014] }\end{array}$ & $\begin{array}{c}\text { Entretenimento } \\
\text { e investigação }\end{array}$ & $\begin{array}{c}\text { localizar } \\
\text { objetos em } \\
\text { um ambiente } \\
2 \text { fixo e } \\
\text { resolver } \\
\text { investigações }\end{array}$ & $\begin{array}{l}\text { diálogos, } \\
\text { foley, 2D } \\
\text { ambiente } \\
\text { e música }\end{array}$ & celular & touchscreen & $\mathrm{X}$ \\
\hline $\begin{array}{c}\text { [Wilhelmsson } \\
\text { et al. 2015] }\end{array}$ & $\begin{array}{c}\text { Entretenimento } \\
\text { e investigação }\end{array}$ & $\begin{array}{c}\text { localizar } \\
\text { objetos em } \\
\text { um ambiente } \\
2 \text { fixo e } \\
\text { resolver } \\
\text { investigações }\end{array}$ & $\begin{array}{l}\text { diálogos, } \\
\text { foley, 2D } \\
\text { ambiente } \\
\text { e música }\end{array}$ & celular & touchscreen & $\mathrm{X}$ \\
\hline $\begin{array}{l}\text { [Rovithis et } \\
\text { al. 2015] }\end{array}$ & $\begin{array}{l}\text { Treinamento e } \\
\text { entretenimento }\end{array}$ & $\begin{array}{l}\text { game da } \\
\text { memória }\end{array}$ & $\begin{array}{c}2 \mathrm{D} \\
\text { ambiente, } \\
\text { efeitos } \\
\text { sonoros }\end{array}$ & computador & teclado & - \\
\hline
\end{tabular}




\begin{tabular}{|c|c|c|c|c|c|c|}
\hline Fonte & Abordagem & Desafio & $\begin{array}{l}\text { Tipo de } \\
\text { som }\end{array}$ & Plataforma & $\begin{array}{c}\text { Interação } \\
\text { com } \\
\text { dispositivo }\end{array}$ & Experimento \\
\hline $\begin{array}{c}\text { [Drossos et } \\
\text { al. 2015] }\end{array}$ & entretenimento & $\begin{array}{l}\text { game da } \\
\text { velha }\end{array}$ & $\begin{array}{c}\text { diálogos, } \\
\text { 3D } \\
\text { ambiente }\end{array}$ & computador & teclado & $\mathrm{X}$ \\
\hline $\begin{array}{l}\text { [Allain et al. } \\
\text { 2015] }\end{array}$ & $\begin{array}{l}\text { Treinamento de } \\
\text { orientação } \\
\text { espacial e } \\
\text { entretenimento }\end{array}$ & $\begin{array}{l}\text { Evitar } \\
\text { obstáculos } \\
\text { sonoros }\end{array}$ & $\begin{array}{c}\text { 3D } \\
\text { ambiente }\end{array}$ & PC & $\begin{array}{c}\text { Joystick } \\
\text { Xbox 360, } \\
\text { Oculus Rift }\end{array}$ & $\mathrm{X}$ \\
\hline $\begin{array}{l}\text { [Oliveira et } \\
\text { al. 2016] }\end{array}$ & $\begin{array}{l}\text { Treinamento de } \\
\text { orientação } \\
\text { espacial com } \\
\text { música }\end{array}$ & $\begin{array}{c}\text { localizar } \\
\text { instrumentos } \\
\text { musicais } \\
\text { pelo som } \\
\text { emitido }\end{array}$ & $\begin{array}{c}\text { 3D } \\
\text { ambiente, } \\
\text { música }\end{array}$ & celular & $\begin{array}{c}\text { sensor } \\
\text { giroscópio }\end{array}$ & $\mathrm{X}$ \\
\hline $\begin{array}{c}\text { [Smith e } \\
\text { Nayar 2018] }\end{array}$ & $\begin{array}{l}\text { Tornar games } \\
\text { de corrida } \\
\text { acessíveis }\end{array}$ & $\begin{array}{l}\text { vencer a } \\
\text { corrida }\end{array}$ & $\begin{array}{c}\text { 3D } \\
\text { ambiente, } \\
\text { música, } \\
\text { efeitos } \\
\text { sonoros, } \\
\text { foley }\end{array}$ & computador & $\begin{array}{c}\text { Joystick, } \\
\text { teclado }\end{array}$ & $\mathrm{X}$ \\
\hline
\end{tabular}

Observa-se na Tabela 1 que a maioria dos games são desenvolvidos para uso no computador. De acordo com Rovithis et al. (2015), este tipo de plataforma dispõe de teclado, que por sua vez, é considerado um dispositivo de entrada acessível à PcD visual, facilitando o controle e interação nos games. Observa-se também, outros métodos de interação com o game, como touchscreen e sensor giroscópio, utilizados no smartphone.

Percebe-se que o uso de áudio games cria sonoramente um Ambiente Virtual completo, permitindo ao jogador obter uma experiência emocional e introspectiva, utilizando-se das técnicas de áudio e interação abordadas anteriormente, possibilitando uma sensação de imersão, enquanto a PcD visual navega pelo game.

\section{Considerações Finais}

Dessa forma, acredita-se que as técnicas de audio games, bem como as técnicas de interação apresentadas, possam ser aplicadas no desenvolvimento de games 3D acessíveis para PcD visual, a partir de computadores e de dispositivos móveis.

Salienta-se que desenvolver pesquisas acerca de mundos virtuais 3D, especialmente acessíveis para $\mathrm{PcD}$ visual, é uma contribuição importante para as áreas de Realidade Virtual, Games e áreas correlatas, que fazem uso de AVs 3D, em diferentes contextos, seja no entretenimento ou na educação. 


\section{Referências}

Allain, K. et al. (2015). An audio game for training navigation skills of blind children. 2015 IEEE 2nd VR Workshop on Sonic Interactions for Virtual Environments, SIVE 2015 - Proceedings, n. March, pp. 49-52.

Balan, O. et al. (2014). Navigational 3D audio-based game-training towards rich auditory spatial representation of the environment. 2014 18th International Conference on System Theory, Control and Computing, ICSTCC 2014, pp. 682-687.

Cuzziol, M. F. (2007). Games 3D: Aspectos de Desenvolvimento. Disponível em: $<$ http://www.teses.usp.br/teses/disponiveis/27/27131/tde-23072009-160753/pt-br.php>.

Drossos, K. et al. (2015). Accessible games for blind children, empowered by binaural sound. Proceedings of the 8th ACM International Conference on Pervasive Technologies Related to Assistive Environments - PETRA'15, pp. 1-8.

Ferreira, F.; Cavaco, S. (2014). Mathematics for all: a Game-Based Learning Environment for Visually Impaired Students. Frontiers in Education Conference (FIE), 2014 IEEE, pp. 1-8.

Horowitz, S.; Looney, S. R. (2014). The Essential Guide to Game Audio: The Theory and Practice of Sound for Games. 254p.

Kim, B. U.; Baek, N. (2014). Developing an audio-based game. 2014 International Conference on IT Convergence and Security, ICITCS 2014, pp. 1-3.

Moher, D.; Liberati, A.; Tetzlaff, J.; Altman, DG. (2009). Preferred reporting items for systematic reviews and meta-analyses: The PRISMA statement. PLoS Medicine, v. 6, n. 7.

Oliveira, P. A. et al. (2016). Virtual stage: An immersive musical game for people with visual impairment. Brazilian Symposium on Games and Digital Entertainment, SBGAMES, pp.135141.

Östblad, P. A. et al. (2014). Inclusive game design. Proceedings of the 9th Audio Mostly on A Conference on Interaction With Sound - AM '14, pp. 1-8.

Pires, D.; Furtado, B.; Carregã, T. (2013). The blindfold soundscape game: a case for participation-centered gameplay experience design and evaluation. Proceedings of the 8 th Audio Mostly Conference, Article No. 9.

Rovithis, E. et al. (2014). Audio games as educational tools: Design principles and examples. 2014 IEEE Games, Media, Entertainment Conference, Toronto, ON, pp. 1-8.

Rovithis, E.; Mniestris, A.; Floros, A. (2014). Educational audio game design. Proceedings of the 9th Audio Mostly on A Conference on Interaction With Sound - AM '14, pp. 1-6. Disponível em: <http://ezproxy.library.nyu.edu:2936/citation.cfm?id=2636879.2636902>.

Simões, D.; Cavaco, S. (2014). An orientation game with 3D spatialized audio for visually impaired children. Proceedings of the 11th Conference on Advances in Computer Entertainment Technology - ACE '14, pp. 1-4.

Smith, B. A.; Nayar, S. K. (2018). The RAD: Making Racing Games Equivalently Accessible to People Who Are Blind. Proceedings of the 2018 CHI Conference on Human Factors in Computing Systems (CHI 2018), pp. 1-12.

Wilhelmsson, U.; Brusk, J.; Ostblad, P. (2015). Accessible Game Culture Using Inclusive Game Design-Participating in a Visual Culture That You Cannot See. 7th International Conference on Games and Virtual Worlds for Serious Applications (VS-Games), Skovde, 2015, pp. 1-8. 